\title{
A Comparative Proteomic Analysis of Erinacine A's Inhibition of Gastric Cancer Cell Viability and Invasiveness
}

\author{
Hsing-Chun Kuo a,b,c,d Yur-Ren Kuo e,f,g Kam-Fai Lee ${ }^{\text {h }}$ Meng-Chiao Hsieh d,i \\ Cheng-Yi Huang ${ }^{d}$ Yung-Yu Hsiehj Ko-Chao Lee ${ }^{k}$ Hsiang-Lan Kuo ${ }^{g}$ Li-Ya Lee \\ Wan-Ping Chen' Chin-Chu Chen' Shui-Yi Tung,
}

\begin{abstract}
a Department of Nursing, Chang Gung University of Science and Technology, Chiayi, ${ }^{\mathrm{b} C h r o n i c}$ Diseases and Health Promotion Research Center, CGUST, Chiayi, 'Research Center for Industry of Human Ecology and Research Center for Chinese Herbal Medicine, College of Human Ecology, Chang Gung University of Science and Technology, Taoyuan, 'Division of Colon and Rectal Surgery, Department of Surgery, Chang Gung Memorial Hospital Chiayi, eDivision of Plastic Surgery, Department of Surgery, Kaohsiung Medical University Hospital, Kaohsiung, ${ }^{\text {fF}}$ aculty of Medicine, College of Medicine, Kaohsiung Medical University, Kaohsiung, '9Department of Biological Sciences, National Sun Yat-Sen University, Kaohsiung,

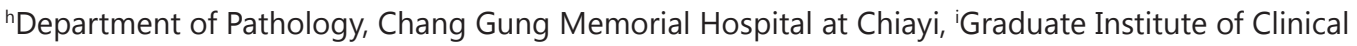
Medical Sciences, College of Medicine, Chang Gung University, Taoyuan, 'Department of HepatoGastroenterology, Chang Gung Memorial Hospital, Chiayi, kDivision of Colorectal Surgery, Department of Surgery, Chang Gung Memorial Hospital, Kaohsiung Medical Center, Chang Gung University College of Medicine, Kaohsiung, 'GRAPE KING BIO Ltd, Taoyuan, ${ }^{m}$ Chang Gung University College of Medicine, Taoyuan, Taiwan
\end{abstract}

\section{Key Words}

Erinacine A • ROS • FAK • p70S6K • 1433S • MTUS2

\begin{abstract}
Background / Aims: Erinacine A, isolated from the ethanol extract of the Hericium erinaceus mycelium, has been demonstrated as a new alternative anticancer medicine. Drawing upon current research, this study presents an investigation of the molecular mechanism of erinacine A inhibition associated with gastric cancer cell growth. Methods: Cell viability was determined by Annexin V-FITC/ propidium iodide staining and migration using a Boyden chamber assay to determine the effects of erinacine $A$ treatment on the proliferation capacity and invasiveness of gastric cancer cells. A proteomic assay provided information that was used to identify the differentially-expressed proteins following erinacine A treatment, as well as the mechanism of its targets in the apoptotic induction of erinacine A. Results: Our results demonstrate that erinacine A treatment of TSGH 9201 cells increased cytotoxicity and the generation of reactive oxygen species (ROS), as well as decreased the invasiveness. Treatment of TSGH 9201 cells with erinacine $A$ resulted in the activation of caspases and the expression of TRAIL. Erinacine A induction of apoptosis was accompanied by sustained phosphorylation of FAK/AKT/p70S6K and the PAK1 pathways, as well as the generation of ROS. Furthermore, the induction of apoptosis and anti-invasion properties by erinacine $A$ could involve the differential expression


of the 14-3-3 sigma protein (1433S) and microtubule-associated tumor suppressor candidate 2 (MTUS2), with the activation of the FAK/AKT/p70S6K and PAK1 signaling pathways. Conclusions: These results lead us to speculate that erinacine A may generate an apoptotic cascade in TSGH 9201 cells by activating the FAK/AKT/p70S6K/PAK1 pathway and upregulating proteins $1433 \mathrm{~S}$ and MTUS2, providing a new mechanism underlying the anti-cancer effects of erinacine $A$ in human gastric cancer cells.

(C) 2017 The Author(s)

Published by S. Karger AG, Basel

\section{Introduction}

Hericium erinaceus, an edible mushroom, has been demonstrated to potentiate the effects of numerous biological activities. H. erinaceus can produce mycelia and biological metabolites, such as erinacines and hericenones, by liquid and solid-state fermentation without harmful effects [1, 2]. Either the H. erinaceus mycelium (erinacines A-I) or its fruit bodies (hericenones $\mathrm{C}-\mathrm{H}$ ) are the source of many bioactive extracts with valuable biological properties [3]. This study has identified that $H$. erinaceus has a number of therapeutic properties, such as antioxidant activity [1], hypolipidemic activity [4], hemagglutinating activity [5], antimicrobial activity [6], antiaging activity [7], and anticancer activity [8, 9]. Erinacine A (Fig. 1)-enriched H. erinaceous mycelia, which exhibit well-known antiinflammatory and anticancer effects [10-13], have been extensively investigated and proposed to promote a decrease in cell viability and enhance apoptosis in various cancer cells. Moreover, our previous studies indicated that $H$. erinaceus mycelium and extracted erinacine A could be used to investigate the in vitro and in vivo antitumor activity through cell cycle arrest in the G1 phase of the human colorectal cancer cells involved in the generation of ROS and the activation of the p70S6K/ NF-kB signaling pathway, which leads to p21 expression and inactivation of cdk2/cyclin E and cdk4/cyclin D1 [12]. However, little is known about the proliferation capacity, the invasiveness of gastric cancer cells, or the mechanism by which erinacine A inhibits cell growth, either by contributing to aggressiveness or cell death, which remains poorly understood.

Gastric cancer, a malignant disease with poor prognosis, ranks as the fourth most frequent cause of malignant tumors in the industrialized world [14]. In a previous study, gastric cancer resulted from an excessive formation of epithelial cells, their ability to evade apoptosis, and their inclination toward tissue invasion and metastasis [15]. Apoptosis is a highly conserved evolutionary process that induces the degradation of unnecessary or damaged cytoplasmic contents in two cascades: the extrinsic and intrinsic pathways, which both lead to the kinase cascade [16]. It is characterized by the emergence and activation of TNFR1/Fas in the plasma membrane and the cleavage of cytosolic BID to truncated tBID by caspase-8, which translocates to the mitochondria via the intrinsic pathway of the kinasesignaling cascade, [17]. Apoptosis can be activated as an important pathway under numerous extra- or intracellular stresses, such as starvation, reactive oxygen species (ROS), anticancer drug stimuli and phenolic phytochemicals $[18,19]$. Recently, we identified that naturally produced erinacine A was reported to induce cell death in various cancer cell lines $[12,13]$ and plays roles in intracellular ROS production, where its levels are elevated in CRC and are sensitive to oxidative damage. It has been suggested that the reorganization of the actin cytoskeleton is a critical cellular response that influences the induction of apoptosis and modulates cell migration via FAK/AKT/p70S6K/ PAK1 kinases [13, 20]. However, whether erinacine A can induce the mechanisms of the apoptotic cascade and its potential effects in gastric cancer cells remains unclear.

Pathological data from a recent study of ours demonstrated that dietary H. erinaceus mycelium and the native erinacine A could exhibit in vitro and in vivo therapeutic effects on cancer invasion and were involved in the phosphorylation of the PI3K/mTOR/ p70S6K and ROCK1/LIMK2/Cofilin pathways. This indicates its possible implication in actin depolymerization and therapy [21], while the activation of various cell membrane receptors, with subsequent triggering of these intracellular signaling cascades, often 


\section{Cellular Physiology Cell Physiol Biochem 2017;43:195-208 \\ \begin{tabular}{l|l|l} 
DOI: 10.1159/000480338 & and Biochemistry Published online: August 29, 2017 & $\begin{array}{l}\text { O 2017 The Author(s). Published by S. Karger AG, Basel } \\
\text { www.karger.com/cpb }\end{array}$
\end{tabular} \\ Kuo et al.: Erinacine A Induces Apoptosis and Inhibition of Metastasis in Gastric Cancer}

involves cytoskeleton rearrangement [22]. In the present study, the protein profile changes of the human gastric cancer cell line TSGH9201 in response to erinacine A treatment were investigated using a proteomics approaches (2DE MS/MS) to explore whether the molecular mechanisms of dietary erinacine A result in anti-cancer activity. Using proteomics approaches, the present study found that native erinacine A administration could induce the protein expression of 1433S and MTUS2, including production of ROS and phosphorylation of FAK/AKT/p70S6K and PAK1, which could be attributed to the inhibition of proliferation, the invasiveness of gastric cancer cells, and the induction of the apoptosis signaling pathway, such as TRAIL and caspases.

\section{Materials and Methods}

Hericium erinaceus Extracts and Analysis of Erinacine $A$

Fresh H. erinaceus dried mycelium (2 kg) was extracted with 95\% ethanol. The extracted ethanol solution was concentrated and fractionated by solvent partition between ethyl acetate and water to afford an $\mathrm{H}_{2} \mathrm{O}$ layer and an EtOAc layer. The EtOAc layer analysis was subjected to silica gel column chromatography according to the previous study [12,13]; HPLC analysis of erinacine A was executed with minor modifications $[12,13]$. The analytical column used was a COSMOSIL 5C18-AR-II $(250 \times 4.6 \mathrm{~mm}$; particle size $5 \mu \mathrm{m}$, Nacalai USA, Inc., Kyoto, Japan). Extraction of $5 \mathrm{mg} / \mathrm{kg} \mathrm{H}$. erinaceus erinacine A with $85 \%$ ethanol was confirmed and quantified by HPLC $[10,11]$. The erinacine A chemical compounds reported in this article (PubChem CID: 10410568) are shown in Fig. 1.

\section{Cell culture}

The human MKN28 and TSGH9201 cells were obtained from the American Tissue Culture Collection (ATCC, USA). Cells were cultured in Dulbecco's modified Eagle's medium (DMEM) supplemented with 10\% fetal calf serum (Gibco), non-essential amino acids, $1 \mathrm{mM}$ sodium pyruvate and $1 \%$ antibiotics (100 units/ $\mathrm{mL}$ penicillin and $100 \mu \mathrm{g} / \mathrm{mL}$ streptomycin). All experiments were performed in plastic tissue culture flasks, dishes or in microplates (Nunc, Naperville, Denmark). Incubation was carried out at $37^{\circ} \mathrm{C}$ in a humidified atmosphere of $5 \% \mathrm{CO}_{2}$ and $95 \%$ oxygen [23], while cells were treated with $10 \mu \mathrm{M}$ erinacine A for $24 \mathrm{~h}$. The cells were collected and washed with PBS and were subsequently transferred to sterile plastic tubes for storage at $-80^{\circ} \mathrm{C}$ until use.

\section{Chemical Reagents and Antibodies}

All culture materials were purchased from Gibco (Grand Island, NY, USA). 3-(4,5-dimethylthiazol2-yl)-2,5-diphenyltetrazolium bromide (MTT), ROS scavenger ( $N$-acetyl cysteine [NAC]), 2,7-dichlorodihydrofluorescein diacetate ( $\left.\mathrm{H}_{2} \mathrm{DCFDA}\right)$, dihydroethidium (DHE), FAK inhibitor (Y15) and mTOR inhibitor (rapamycin) were purchased from Sigma (St. Louis, MO, USA). Mouse monoclonal antibodies against TRAIL, caspase 8, Bcl-2, Bcl-XL, cytochrome c, caspase 9 and caspase 3, Fas-L, t-Bid, cytochrome c, nucleophosmin (NPM), 14-3-3 protein zeta/delta (1433Z), 14-3-3 protein sigma (1433S), microtubuleassociated tumor suppressor candidate 2 (MTUS2), $\beta$-actin and horseradish peroxidase-linked anti-rabbit or mouse IgG were purchased from Santa Cruz Biotechnology (Santa Cruz, CA, USA). Rabbit polyclonal antibodies against phosphorylated FAK Tyr ${ }^{579 / 577}$, AKT Ser ${ }^{473}$, p70S6K $\mathrm{Thr}^{389}$, and PAK1 $\mathrm{Thr}^{423}$, were purchased from Cell Signaling Technology (Beverly, MA, USA). SDS, NP-40, sodium deoxycholate, protease inhibitor cocktails were purchased from Sigma.

Measurement of cell viability and reactive oxygen species

Cell viability, as previously reported by Annexin V-FITC/ propidium iodide staining (Biosource International, USA), was used to quantify the percentage of cells undergoing apoptosis. The intracellular accumulation of ROS (02-) was determined by the H2DCFDA (2, 7-dichlorodihydrofluorescein diacetate) fluorescent probes, and the cells were washed prior to FACS analysis and analysis by Cell Quest software (Becton Dickenson), which was previously described [12]. The results are presented as a percentage of the fluorescent intensity compared with the control sample. The apoptotic cells (V+/PI-) were measured by the fluorescence-activated cell sorter analysis in a FACS analyzer (Becton Dickinson). The data represent three independent experiments. 


\section{Cellular Physiology Cell Physiol Biochem 2017;43:195-208 \\ \begin{tabular}{ll|l} 
and Biochemistry Published online: August 29, 2017 & $\begin{array}{l}\text { (C) } 2017 \text { The Author(s). Published by S. Karger AG, Basel } \\
\text { www.karger.com/cpb }\end{array}$
\end{tabular} \\ Kuo et al.: Erinacine A Induces Apoptosis and Inhibition of Metastasis in Gastric Cancer}

\section{Matrigel invasion assay}

The Boyden chamber assay used for the analysis of tumor cell invasion is based on a chamber with two medium-filled compartments as previously described [19]. Cells $\left(1 \times 10^{5} / \mathrm{ml}\right)$ in serum-free medium were added to an inner cup of the 48-well Transwell chamber (Corning Life Sciences, Corning, New York) that had been coated with $50 \mu \mathrm{l}$ of Matrigel (BD Biosciences, Franklin Lakes, New Jersey; 1:10 dilution in serum-free medium). Medium supplemented with $10 \%$ serum or the indicated agent was added to the outer cup. Cells were allowed to migrate for $24 \mathrm{~h}$ at $37^{\circ} \mathrm{C}$ in a humidified atmosphere containing $5 \% \mathrm{CO}_{2}$. The membrane was fixed and stained with modified Giemsa stain (Sigma-Aldrich). The human MKN28 and TSGH9201 cells on the lower side of the membrane were counted using a light microscope at $200 \times$ magnification. The number of cells that migrated to the lower side of the membrane was determined [13].

Two-dimensional protein electrophoresis analysis of the proteomic profiles of TSGH9201 cells and image analysis

Total proteins were extracted from Erinacine A-treated and untreated TSGH9201 cells by incubation with lysis buffer. The protein pellets were re-solubilized in rehydration solution and kept at $-80^{\circ} \mathrm{C}$ until further analysis. The chemicals and reagents used for 2D gel electrophoresis were described before [24]. The total amount of proteins was determined using the Bradford assay with bovine serum albumin as the standard sample for normalization. Following cell lysis, the total cell protein was precipitated with $10 \%$ trichloroacetate in acetone. Protein samples were suspended in rehydration solution and subjected to isoelectric focusing (IEF) in 13-cm immobilized ReadyStrip ${ }^{\mathrm{TM}}$ (Bio-Rad, Hercules, CA) and IPG Strips (pH 3-10) in an Ettan IPGphor II apparatus (Amersham Biosciences). The second dimension of electrophoresis was carried out using 10\% SDS-PAGE gels. Then, BioSafe Coomassie stain was added, and the gels were stained overnight.

In-gel digestion and identification of peptide fingerprints using MALDI-TOF/TOF

Six pairs of silver-stained 2D SDS-PAGE gels in which total cell proteins had been resolved were scanned using ImageMaster 2D Platinum Software 6.0 (Amersham Biosciences), which was utilized for spot detection and matching of each pair of silver-stained gels. The quantity of each spot was recorded and compared, and the protein spots that differed by at least 3-fold among all six pairs of 2D gels were subjected to in-gel digestion for matrix-assisted laser resorption ionization-time-of flight/time-of-flight (MALDI-TOF/ TOF) mass spectrometric analysis with a MALDI-LC-ESI configuration. The gel pieces were then dehydrated and subjected to trypsin digestion. Mass spectra were acquired as the sum of the ion signals. The peptide fingerprints were selected in the range of 700-4000 Daltons and then analyzed by using the MASCOT search engine (http://www.matrixscience.com). Search parameters were defined as follows: Database, Swiss-Prot; taxonomy, Homo sapiens; enzyme, trypsin. A Mascot score of $P<0.05$ was considered statistically significant as described. MALDI-TOF/TOF data were searched in-house using the MASCOT software (ver 2.2.04). The protein identifications required for the detection of unique peptides and proteins with more than two spectral counts were selected for further analysis with their MW and pI consistent with the gel regions. Proteins identified with a higher MASCOT score in the bovine database than in the human database were considered to be serum contamination and were removed $[13,24]$.

\section{Preparation of total cell extracts and immunoblot analyses}

Cells were lysed with RIPA lysis buffer (50 mM Tris-HCl (pH 7.4), 1\% NP-40, 0.25\% Na-deoxycholate, $150 \mathrm{mM} \mathrm{NaCl}, 1 \mathrm{mM}$ EDTA, $1 \mathrm{mM}$ PMSF, $1 \mathrm{mg} / \mathrm{ml}$ Aprotinin, $1 \mathrm{mM} \mathrm{Na} \mathrm{VO}_{4}, 1 \mathrm{mM} \mathrm{NaF}$ ). The total cell lysate (50 $\mu \mathrm{g}$ of protein) was separated by SDS-polyacrylamide gel electrophoresis (PAGE) (12\% running, $4 \%$ stacking) and analyzed using the designated antibodies and the Western Lighting chemiluminescent detection system (Bio-Rad, Hercules, CA), as previously described [12].

\section{Statistical Analysis}

Data are reported as the mean \pm standard deviation (SD) of three independent experiments and were analyzed by one-way analysis of variance (ANOVA). The data were analyzed using the SAS statistical software package "SigmaPlot," version 9.0 (SAS Institute Inc., Cary, NC, USA) [13]. 


\section{Cellular Physiology Cell Physiol Biochem 2017;43:195-208



\section{Results}

Erinacine A increased the apoptotic body formation and anticancer invasiveness of gastric cancer cells

To verify erinacine A-induced cell toxicity, this study examined the changes in TSGH9201 cell morphology. As shown in Fig. 1A and 1B, exposure to erinacine A $(10 \mu \mathrm{M})$ for $24 \mathrm{~h}$ caused the accumulation of cytoplasmic vacuoles in the cells, as well as cell shrinking, rounding, and detachment. The effect of various concentrations of erinacine $A(1-10 \mu \mathrm{M})$ on the percentage of annexin V-positive cells was assayed and was shown to be $13 \%$. The invasiveness and motility of MKN28 and TSGH9201 cells was determined by Boyden chamber assay following treatment with high concentrations of erinacine A (up to $10 \mu \mathrm{M}$ ). The results suggested that there was reduced Matrigel invasion in a dose-dependent manner, with an inhibitory concentration at $50 \%$ of the control value of $10 \mu \mathrm{M}$ (Fig. 1C) for $24 \mathrm{~h}$. Erinacine A initially increased the generation of superoxide in TSGH9201 cells after $6 \mathrm{~h}$, compared to the control group (Fig. 1D), and was examined using a DCFDA probe. Based on this concentration range, to investigate whether erinacine A can induce apoptosis and anti-invasion potential involving the signaling pathway, this study assayed whether H. erinaceus mycelium erinacine A provides substantial therapeutic advantages via TSGH9201 cells.

Apoptotic induction by erinacine A in gastric cancer TSGH9201 cells

To determine the effects of erinacine A-induced apoptosis on the persistent extrinsic apoptosis pathway via activation of the caspase cascade in TSGH9201 cells, this study used kinetic studies to evaluate TRAIL, caspase 8 , and Bcl-2, Bcl-XL expression, as well as caspase 9 and caspase 3, during a $24 \mathrm{~h}$ period. As shown in Fig. 2, erinacine A is shown to cause detectable and increased levels of TRAIL and cytochrome $\mathrm{c}$ in the cytosol, as well as a timedependent cleavage of Bcl-2 and Bcl-XL for 24 h. Treatment with erinacine A resulted in the proteolytic processing of caspase 9 and caspase 3 (17-36 kDa) into several smaller subunits. It is clearly shown that erinacine A induced the activation of caspase- 3 and the release of cytochrome c, both of which are hallmarks of apoptosis (Fig. 2).

2-DE profiling of the differentially expressed proteins of TSGH9201 gastric cancer cells treated with erinacine $A$

Proteomic technologies can be used to screen the target molecules of erinacine A activities, identify new protein expression profiles, and elucidate the underlying mechanisms of erinacine A effects. In our proteomic study of TSGH9201 cells, the lysates were prepared, and the protein spots were then visualized. Figure 2 illustrates that seventeen differences were observed after erinacine A treatment in the silver-stained 2D-PAGE analysis, including downregulation of 4 proteins and upregulation of 13 proteins by erinacine A. Regarding image analysis, protein expression profile gels (six pairs from the control and the erinacine A-treated groups) were compared using image analysis (ImageMaster software) (Fig. 4). The expression level of the total protein displays is shown with a p-value $<0.05$, as determined by Student's t-test, and reproducible changes in intensity of more than two-fold (compared to the untreated control), which were analyzed by MS/MS spectrometry in the erinacine A-treated group. There were 17 locations that were subjected to peptide fingerprint identification using MALDI-TOF MS. Using proteomics approaches, Table 1 presents the close-up views of a representative gel and displays several differentially expressed apoptosis-related proteins of the erinacine A target, which inhibits invasive action in TSGH9201 cells.

Validation of the differentially displayed proteins in the erinacine A-treated TSGH9201 cells

Locations 3, 4, 14, and 16 were subsequently identified using 2D proteomic analysis, including 14-3-3 protein "zeta/delta (1433Z) and nucleophosmin (NPM), as well as microtubule-associated tumor suppressor candidate 2 (MTUS2) and 14-3-3 protein sigma (1433S). This study identified these proteins using western blotting assays, which 


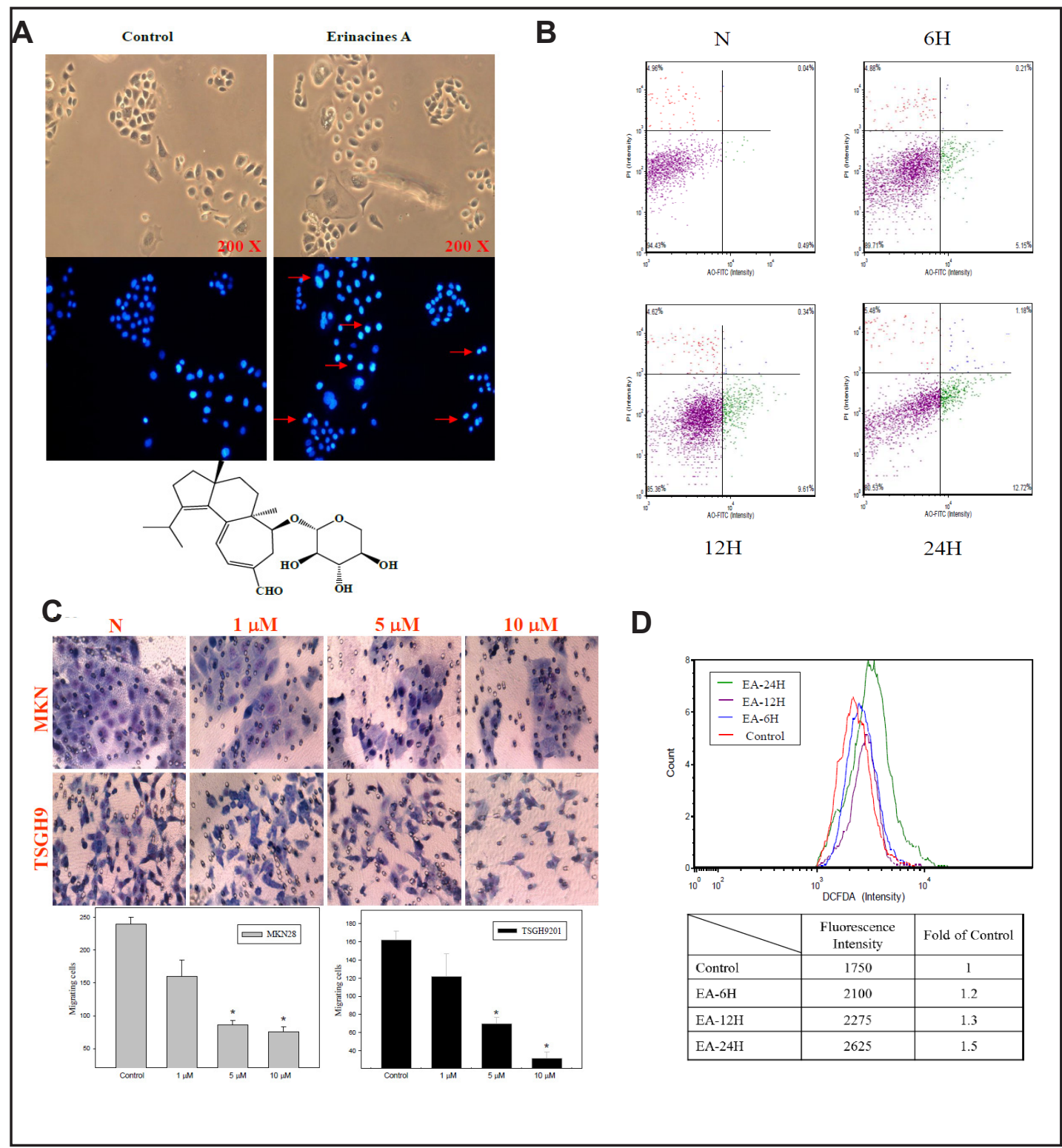

Fig. 1. Effects of erinacine A on cell viability, the morphological characteristics of human gastric cancer cells, and its role in assessing cell death. (A) TSGH 9201 cells were treated with either $0.1 \%$ DMSO (as control) or erinacine $\mathrm{A}(1-10 \mu \mathrm{M})$ for $24 \mathrm{~h}$, and the proportion of surviving cells was measured by DAPI staining. Apoptotic cells were measured using fluorescence microscopy. (B) After an indicated treatment of $24 \mathrm{~h}$, the TSGH 9201 cells were stained with FITC-conjugated Annexin-V and PI for flow cytometry analysis as described in the 'Materials and methods'. The percentages presented in each frame depict the apoptotic cells. (C) Effect of erinacine A on the invasiveness of MKN28 and TSGH9201 cells. Cells were incubated with various concentrations of erinacine A for $24 \mathrm{~h}$. Invasion through a layer of Matrigel was determined by the Boyden Chamber method as described in the 'Materials and methods'. The lower and upper chemotaxis cells were separated by a polycarbonate membrane. Microscopy images detected cells that migrated into the inner membrane, at a 200X magnification. The cell migration was quantified by counting the number of cells that migrated into the inner membrane. Control cells

are consistent with the 2-DE results, as shown in Fig. 5A. Of these, $1433 \mathrm{Z}$ and NPM were downregulated, while 1433S and MTUS2 were upregulated in erinacine A-treated cells, which may mediate cancer recurrence and regulate the apoptosis effects of erinacine $A$ in TSGH9201 cells [25-28]. 


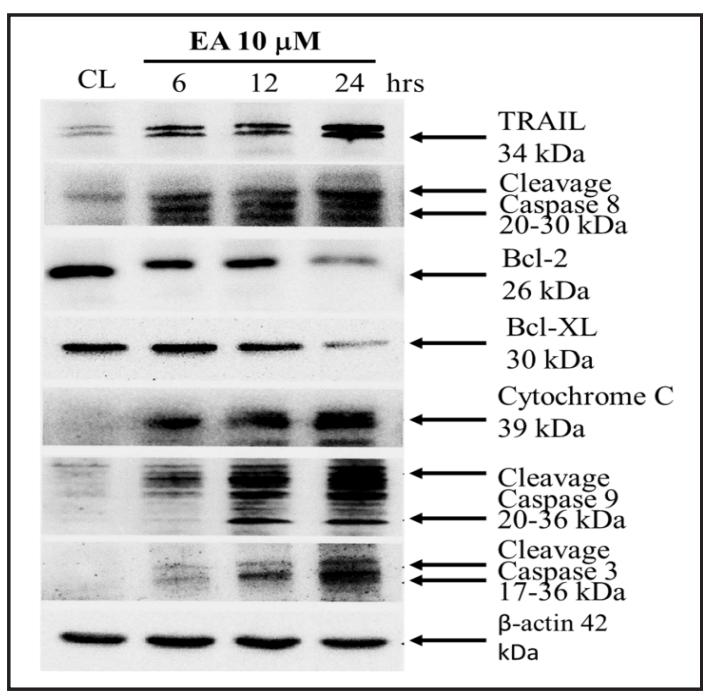

Fig. 2. Effect of erinacine A on TRAIL, caspase 8, Bcl-2, Bcl-XL, cytochrome c, caspase 9 and caspase 3. Cells were treated with erinacine A for 0-24 h, were separated on a $15 \%$ SDS-PAGE gel, and subsequently immunoblotted with antibodies against TRAIL, cleaved caspase $8, \mathrm{Bcl}-2$, Bcl-XL, cytochrome c, cleaved caspase 9 and cleaved caspase 3, or b-actin, which served as an internal control. Erinacine A induced the translocation of cytochrome c. Equal amounts of protein from the cytosolic fraction of TSGH9201 cells were treated with $10 \mathrm{mM}$ erinacine A.

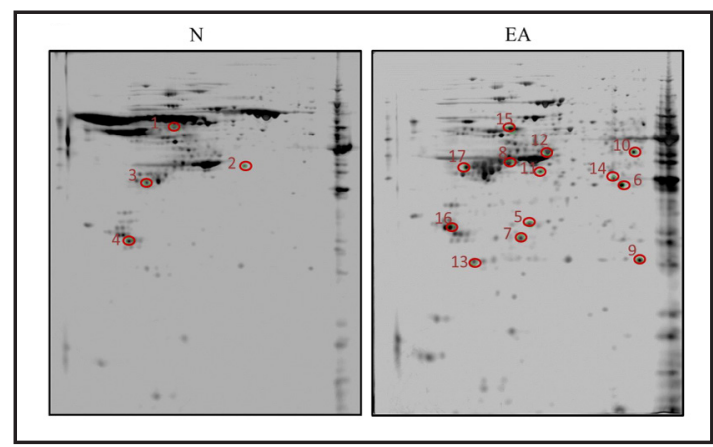

Fig. 3. Representative two-dimensional gel electrophoretograms of erinacine A treatment of TSGH 9201 cells. Six pairs of cell protein extracts were evaluated, and a representative pair of proteomic gel images from the erinacine A-treated and untreated groups are shown. Ten groups of 15 protein spots with a threefold difference between both groups were subjected to MALDI-TOF-TOF analysis. Five protein spots with a significant decrease compared with the untreated group were encircled and annotated. Five spots with a significant increase in the erinacine A group were reported. The full names of these differentially displayed protein spots are listed in Table 1.

Table 1. Differentially expressed protein (N> EA)

\begin{tabular}{|c|c|c|c|c|c|}
\hline Spot & Protein name & $\mathrm{Mr} / \mathrm{PI}$ & Accession no & $\begin{array}{l}\text { MASCOT } \\
\text { score }\end{array}$ & $\begin{array}{l}\text { Matched } \\
\text { peptides }\end{array}$ \\
\hline 1 & Rab GDP dissociation inhibitor alpha & $51 / 4.8$ & GDIA_HUMAN & 87 & 3 \\
\hline 2 & COP9 signalosome complex subunit 4 & $46 / 5.4$ & CSN4_HUMAN & 22 & 7 \\
\hline 3 & 14-3-3 protein zeta/delta & $27 / 4.5$ & 1433Z_HUMAN & 482 & 30 \\
\hline 5 & Chloride intracellular channel protein 1 & $27 / 4.9$ & CLIC1_HUMAN & 349 & 17 \\
\hline 6 & $60 S$ acidic ribosomal protein & $34 / 5.6$ & RLA0_HUMAN & 585 & 23 \\
\hline 7 & Ran-specific GTPase-activating protein & $23 / 5.0$ & RANG_HUMAN & 351 & 28 \\
\hline 8 & TPR and ankyrin repeat-containing protein 1 & $340 / 6.3$ & TRNK1_HUMAN & 48 & 6 \\
\hline 11 & Glutaredoxin-3 & $37 / 5.1$ & GLRX3_HUMAN & 149 & 10 \\
\hline 12 & Eukaryotic initiation factor $4 \mathrm{~A}-\mathrm{I}$ & $46 / 5.1$ & IF4A1_HUMAN & 68 & 1 \\
\hline 13 & Translationally-controlled tumor protein & $19 / 4.6$ & TCTP_HUMAN & 387 & 22 \\
\hline 14 & $\begin{array}{l}\text { Microtubule-associated tumor suppressor } \\
\text { candidate } 2\end{array}$ & $150 / 6.2$ & MTUS2_HUMAN & 44 & 5 \\
\hline 15 & Tubulin alpha-1B chain & $50 / 4.8$ & TBA1B_HUMAN & 1095 & 82 \\
\hline 16 & $14-3-3$ protein sigma & $27 / 4.5$ & 1433S_HUMAN & 807 & 68 \\
\hline 17 & 40 S ribosomal protein SA & $32 / 4.6$ & RSSA_HUMAN & 1034 & 113 \\
\hline
\end{tabular}




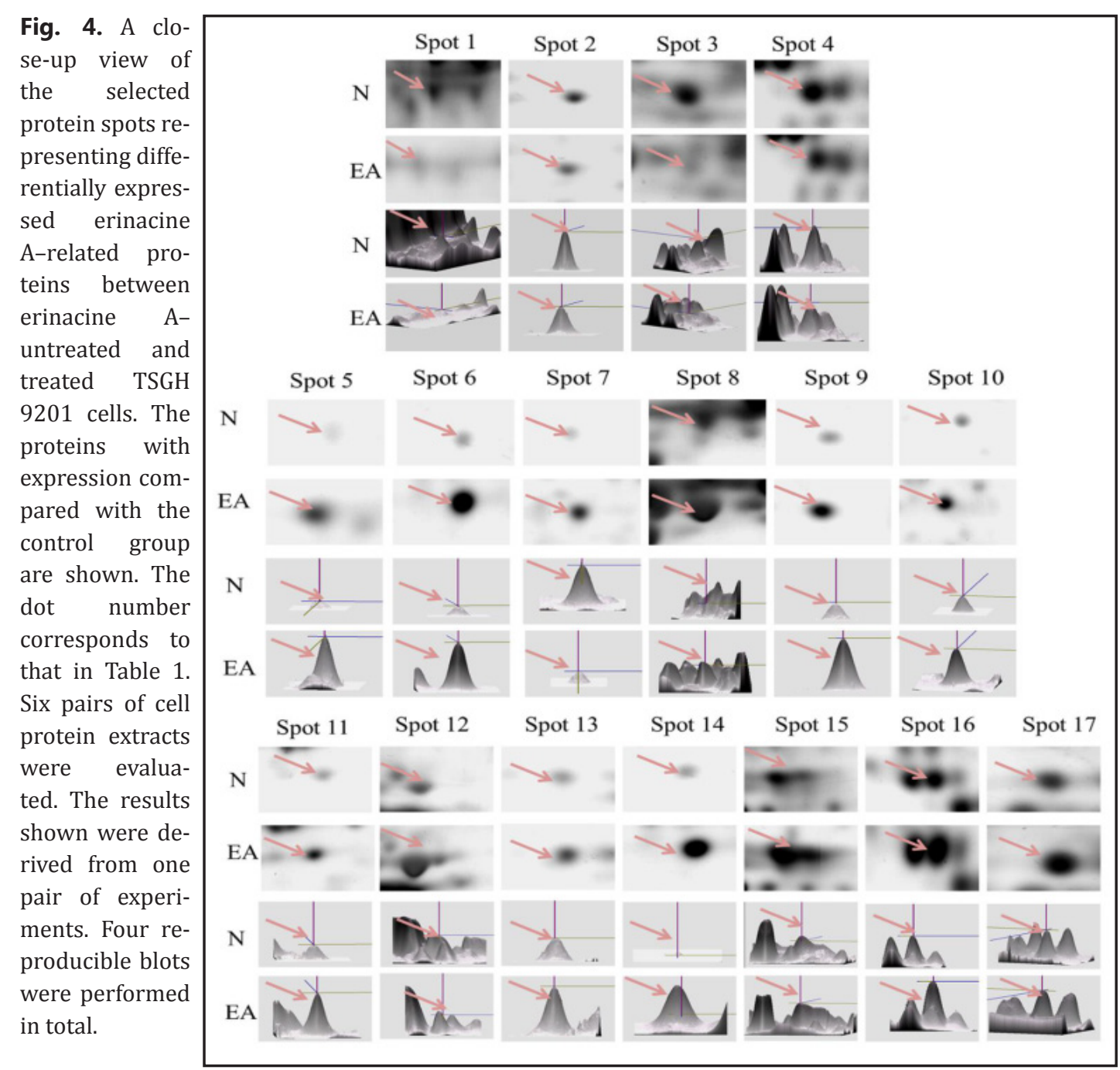

Erinacine A can upregulate levels of $1433 S$ and MTUS2 and the FAK/AKT/p70S6K and PAK1 signaling mechanism, as well as ROS generation

Our previous study suggested that erinacine A could inhibit the growth of colorectal cancer both in vitro and in vivo, which could be attributed to the inhibition of proliferation and the induction of the apoptosis signaling pathway, such as on the generation of ROS via activated PI3K/mTOR/p70S6K and ROCK1/LIMK2/Cofilin pathways. This could be a good molecular strategy for the cancer-related actin depolymerization pathway [20]. Proteomic analyses confirmed that erinacine A decreases cell growth and the aggressive status of TSGH9201 gastric cancer cells via the interaction between ROS and the phosphorylation of FAK/AKT/p70S6K and PAK1 from 2D [21]. This finding, as presented in Fig. 5B, shows significantly increased total lysate protein with the phosphorylation of FAK, AKT, p70S6K, PAK1 compared with the control (CL) after being induced with the indicated dose of erinacine A after $6 \mathrm{~h}$. This is often accompanied by the activation of an abundance of proteins, detected by $2 \mathrm{D}$ proteomic analysis, as the mechanism that decreases cell growth and the aggressive status of TSGH9201 cells. As shown in Table 2, the erinacine A-induced cell death values were $15 \%, 2 \%, 3 \%$ and $2 \%$, respectively $\left({ }^{*} P<0.01\right)$, and the cells' migration levels were reduced to $30 \%, 68 \%, 64 \%$, and $58 \%\left({ }^{*} P<0.01\right)$. They were reversed after pretreatment with NAC (ROS scavenger), Y15 (FAK Inhibitor), and Rapa (mTOR inhibitor), indicating a decrease in the induced cell viability and aggressive status in the TSGH9201 cells.

\section{KARGER}




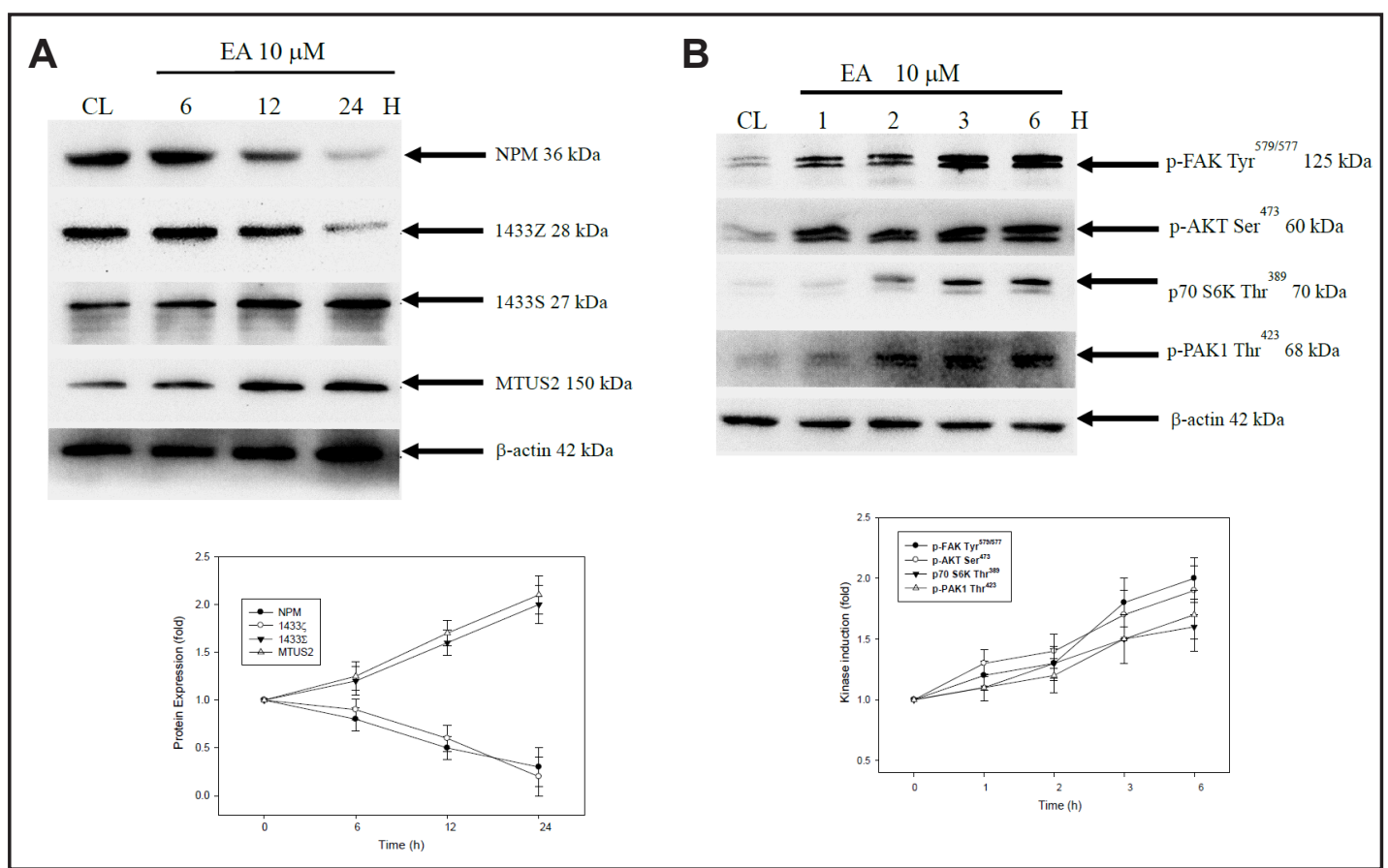

Fig. 5. Validation of the identified differential proteins and activation of FAK/AKT/p70S6K and PAK1 pathways between erinacine A-untreated (CL) and treated (EA) TSGH 9201 cells. (A) Data presented on the Western blot were derived from a representative study, and comparisons of protein expression were calculated from three replicate experiments. The proteins with lower expression (NPM and 1433Z) and higher expression (1433S and MTUS2) in erinacine A-treated cells are shown for the indicated times. (B) The effects of erinacine A on protein levels of phosphorylated FAK, AKT, p70S6K, PAK1 and b-actin were detected with the indicated antibodies.

Reactive oxygen species and the FAK/ AKT/p70S6K/PAK1 signaling pathway contributed to the erinacine A-induced levels of 14335 and MTUS2, as well as decreased cell death and aggressiveness in TSGH9201 cells

Previous studies demonstrated that erinacine A-mediated apoptosis and antiaggressive status are involved in the actin depolymerization pathway. In addition, recent research has shown that several partners of PAK are able to phosphorylate or activate the MAP kinases. PI3-kinase/Akt
Table 2. Effects of the kinase inhibitor on the erinacines A induction associated with cell death and aggressive status in TSGH 9201 cells

\begin{tabular}{lcc}
\hline & $\%$ of cell death & Migration (\%) \\
\hline Control & 0.5 & 100 \\
Erinacines A & 15 & 30 \\
Erinacines A NAC & 2 & 69 \\
Erinacines A Y15 & 3 & 64 \\
Erinacines A Rapa & 2 & 58 \\
\hline
\end{tabular}
and LIM kinases have been proposed in cytoskeleton regulation $[29,30]$. This study detected two proteins, 1433S and MTUS2, referring to TIP150, which had previously been identified as a tumor suppressing protein regulator of the signal transduction pathways associated with the control of cell proliferation, differentiation, and survival, as well as the regulation of microtubule plus-end dynamics [31]. To confirm the association of erinacine A's action in the differential expression of the 1433S and MTUS2 proteins, as well as the activation of the FAK/AKT/p70S6K and PAK1 signaling pathways, which either contribute to cell death or cell motility, this study investigated whether the effects of kinase inhibitors would cause resistance to erinacine A-induced signaling pathways. As shown in Fig. 5, NAC, Y15, and Rapa almost blocked the erinacine A-induced expression of 1433S and MTUS2, as well as the phosphorylation of FAK/AKT/p70S6K and PAK1, compared with the erinacine A treatment 


\section{Cellular Physiology Cell Physiol Biochem 2017;43:195-208 \\ \begin{tabular}{ll|l} 
and Biochemistry & $\begin{array}{l}\text { DOI: 10.1159/000480338 } \\
\text { Published online: August 29, } 2017\end{array}$ & $\begin{array}{l}\text { (c) } 2017 \text { The Author(s). Published by S. Karger AG, Basel } \\
\text { www.karger.com/cpb }\end{array}$ \\
\hline Kun
\end{tabular}}

at $6 \mathrm{~h}$. Differentially displayed proteins were assayed and validated from all the tumor sections. Herein, the effects of erinacine A treatment-induced apoptosis and anti-aggressive pathways in TSGH9201 cells were detected via the ROS trigger and continuously through the FAK/AKT/p70S6K and PAK1 signaling pathways.

\section{Discussion}

Gastric cancer is one of the most common causes of cancer-related death worldwide [32], especially in East Asian countries, such as China and Japan. Various drugs have been intensively researched in many animal experiments and clinical trials for gastric cancer [33, 34]. Some studies have indicated that chemopreventive compounds in dietary foods are a potential source of safe bioactive compounds for cancer chemoprevention and suppression of proliferation via the primary target of ROS that damages DNA and the activation of signaling pathways, as well as the expression of proteins involved in the induction of apoptosis [12, $13,19]$. It is well known that ROS, a class of oxygen-containing and active species, have an important effect on a series of cellular programs, including apoptosis, and are believed to activate signaling pathways via the activation of tyrosine kinases and tyrosine phosphatases. Rho-GTPase, PI3K, AKT, p70S6K, and MAP kinases target the antioxidant-induced cancer cell toxicity of phytochemicals [18]. This study demonstrated for the first time that erinacine A, when isolated from the Hericium erinaceus mycelium, inhibited the viability and invasiveness of gastric cancer cells, including MKN28 and TSGH 9201 cells. Additionally, ROS levels and activation of the FAK/AKT/p70S6K/PAK1 signaling pathway were determined (Fig. 1 and 5B). We also found that the erinacine A induced accumulation of TSGH 9201 cells in a timedependent manner with the induction of apoptosis (Fig. 1), similar to other MKN28 cells (unpublished data). Treatment with erinacine A resulted in the activation of TRAIL, caspase 8, caspase 9 and caspase 3 and the release of cytochrome c, whereas there was a decrease in the cellular levels of Bcl-2 and Bcl-XL in erinacine A-induced apoptosis (Fig. 2). Moreover, the naturally extracted erinacine A-induced cell viability and aggressive status decrease could change remarkably after pretreatment with NAC and Y15. On the contrary, Rapa kinase inhibitors reversed erinacine A-induced TSGH 9201 cell apoptosis and led to a reduction in cancer cell migration in vitro (Table 2). Herein, this study suggested a necessary role for the FAK/AKT/p70S6K and PAK1 pathways during the triggering of apoptosis and anti-invasion by erinacine $\mathrm{A}$.

The medicinal mushroom, Hericium erinaceus, is widely consumed in Asian countries for its nutritional and health benefits, which are reported to be both medicinal and important in inhibiting the metastasis of cancer cells [35]. Many studies have demonstrated that Cui et al. isolated a novel glycoprotein from $H$. erinaceus mycelia cultures, which inhibited human gastric cancer cells by promoting apoptosis and cell cycle arrest at the S-phase. The same extracts are reported to prevent gastric ulcers, chronic gastritis, and other digestive tractrelated diseases [36]. However, the biochemical targets of the H. erinaceus mycelium ethanol extract, erinacine $A$, on the anticarcinogenic properties of gastric cancer cells and the mechanism by which $H$. erinaceus mycelium and erinacine A induces cells apoptosis remain poorly understood. Our previous data demonstrated the mechanisms of colorectal cancer cell inhibition and activation of the PI3K/AKT/p70S6K and actin depolymerization pathways by erinacine A. To validate these findings in gastric cancer, a further study using a 2-DE-based proteomic analysis is needed to determine whether there are cytoskeleton-mediated actions. Microtubule-associated tumor suppressor candidate 2 (MTUS2) and 14-3-3 protein sigma (1433S) were more abundant in erinacine A induction, whereas 14-3-3 protein zeta/delta (1433Z) and nucleophosmin (NPM) were at lower levels (Fig. 3, 4, and 5A) [25, 26]. Many studies have suggested that $1433 \mathrm{Z}$ overexpression, as found in multiple cancers, participated in essential cellular processes, such as signal transduction, apoptosis, and cell migration, with poor prognosis in cancer patients [25]. We have investigated the overexpression or mutation of NPM in human cancer cells as a prognostic marker and therapeutic target, as 
well as their prognostic impact on patients with primary gastric cancer [37]. Erinacine A-mediated upregulation of the tumor suppressor proteins MTUS2 and 1433S is thought to be associated with the antitumor activity of erinacine A in gastric cancer cells. Recent studies showed that $1433 \mathrm{~S}$ seems to be directly involved in human cancer that is associated with $\mathrm{G} 2 / \mathrm{M}$ checkpoint control in the cell cycle $[27,38]$. In addition, MTUS2 plays a central role in controlling the microtubule plus-end-tracking protein by regulating cell division and migration through its mitotic centromere-associated kinesin, a microtubule depolymerase [28, 31]. The cytoskeleton depolymerization pathway has been recognized as a critical cellular response that controls the apoptosis and inhibition of cell migration-activated Rho GTPase through its effector kinases: ROCK1 and ROCK2 [39, 40]. Based on the proteomic displays of TSGH9201 cells, these results showed important findings, meaning that the phosphorylation of the FAK/AKT/ p70S6K and PAK1 pathways results in the downstream expression of the MTUS2 and 1433S genes, as well as the execution of apoptosis and anti-invasiveness by erinacine A (Fig. 6). Our findings are in full agreement with previous study reports demonstrating that steroids hormones, including glucocorticoids and androgens, are important in controlling various cellular functions, such as the apoptotic responses and migratory potential in membrane hormone receptorstimulated FAK/PI3K/p70S6K and PAK1 pathways in various human cancer cells [41-43]. This effect most likely reflects the formation of a cytoskeleton rearrangement resulting in erinacine A-dependent reduction of cell motility.

In conclusion, this study demonstrated that erinacine $\mathrm{A}$, as found in the Hericium erinaceus mycelium, induces apoptosis in gastric cancer cells and that the generation of ROS is critical for erinacine A-induced morphological characteristics, such as cell rounding, apoptosis, and inhibition of metastasis in gastric cancer. Based on the proteomic differential proteins, this study suggests that the upregulation of $1433 \mathrm{~S}$ and MTUS2 expression by erinacine A is mediated by actin cytoskeleton reorganization, which is controlled by the ROS and FAK/



Fig. 6. Effects of the kinase inhibitors blocking erinacine A-induced ROS generation and activation of FAK/AKT/p70S6K and PAK1 pathway-related proteins. Kinase inhibitors were treated either with or without erinacine A in TSGH 9201 cells after 1 h. All cell lysates were prepared and subjected to Western blot analysis. Protein levels of phosphorylated FAK, AKT, p70S6K and PAK1, as well as 1433S, MTUS2 and $\mathrm{b}$-actin, were detected with the indicated antibodies. The independent experiments were conducted and showed the same pattern of change as the levels of the representative experiment shown here.

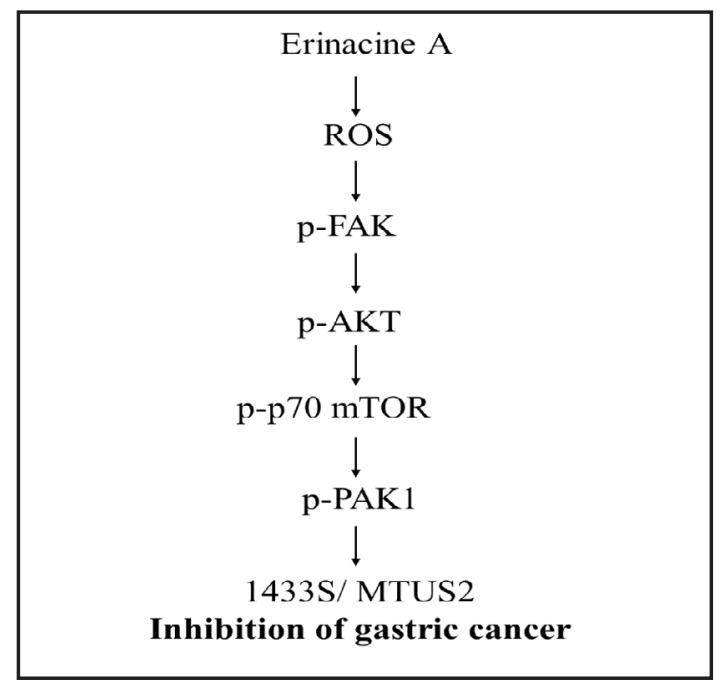

Fig. 7. Schematic presentation of the signaling pathways involved in erinacine A-inhibited cell invasion in TSGH 9201 cells. The effect of erinacine A on the production of ROS-activated phosphorylation of FAK/AKT/p70S6K and PAK1, as well as 1433S and MTUS2 pathways, which induce the $1433 \mathrm{~S}$ and MTUS2 upregulation and result in inhibition of TSGH 9201 cell growth. 


\section{Cellular Physiology Cell Physiol Biochem 2017;43:195-208 \\ \begin{tabular}{ll|l} 
DOI: 10.1159/000480338 & and Biochemistry & $\begin{array}{l}\text { O 2017 The Author(s). Published by S. Karger AG, Basel } \\
\text { www.karger.com/cpb }\end{array}$ \\
\hline
\end{tabular}

AKT/p70S6K/PAK1 signaling pathways (Fig. 7). This study provides additional evidence that erinacine A can inhibit metastatic activity in gastric cancer cells as a new alternative medicine for anticancer effects.

\section{Acknowledgments}

Funding for this study was provided by research grants (CMRPF6F0031, CMRPF6G0011, CMRPG6D0243, CMRPG6D0242, and CMRPG6D0241) from the Chang Gung Memorial Hospital, Chia-Yi. This study was also supported by the BMRPD42, CLRPG8D0112 and by the Ministry of Science and Technology, Taiwan (MOST 103-2622-B-255-001-CC3 and MOST 103-2313-B-255-001 and MOST 104-2320-B-255 -003 -MY3).

\section{Disclosure Statement}

The authors confirm that there are no conflicts of interest.

\section{References}

1 Malinowska E, Krzyczkowski W, Lapienis G, Herold F: Improved simultaneous production of mycelial biomass and polysaccharides by submerged culture of Hericium erinaceum: optimization using a central composite rotatable design (CCRD). J Ind Microbiol Biotechnol 2009;36:1513-1527.

-2 Ulziijargal E, Mau JL: Nutrient compositions of culinary-medicinal mushroom fruiting bodies and mycelia. Int J Med Mushrooms 2011;13:343-349.

-3 Friedman M: Chemistry, Nutrition, and Health-Promoting Properties of Hericium erinaceus (Lion's Mane) Mushroom Fruiting Bodies and Mycelia and Their Bioactive Compounds. J Agric Food Chem 2015;63:71087123.

-4 Yang BK, Park JB, Song CH: Hypolipidemic effect of an Exo-biopolymer produced from a submerged mycelial culture of Hericium erinaceus. Biosci Biotechnol Biochem 2003;67:1292-1298.

5 Gong M, An J, Lu HZ, Wu CF, Li YJ, Cheng JQ, Bao JK: Effects of denaturation and amino acid modification on fluorescence spectrum and hemagglutinating activity of Hericium erinaceum Lectin. Acta Biochim Biophys Sin (Shanghai) 2004;36:343-350.

6 Yim MH, Shin JW, Son JY, Oh SM, Han SH, Cho JH, Cho CK, Yoo HS, Lee YW, Son CG: Soluble components of Hericium erinaceum induce NK cell activation via production of interleukin-12 in mice splenocytes. Acta Pharmacol Sin 2007;28:901-907.

7 Shimbo M, Kawagishi H, Yokogoshi H: Erinacine A increases catecholamine and nerve growth factor content in the central nervous system of rats. Nutrition Res 2005;25:617-623.

-8 Lee JS, Hong EK: Hericium erinaceus enhances doxorubicin-induced apoptosis in human hepatocellular carcinoma cells. Cancer Lett 2010;297:144-154.

-9 Li G, Yu K, Li F, Xu K, Li J, He S, Cao S, Tan G: Anticancer potential of Hericium erinaceus extracts against human gastrointestinal cancers. J Ethnopharmacol. 2014;153:521-530.

$>10$ Lee KF, Chen JH, Teng CC, Shen CH, Hsieh MC, Lu CC, Lee KC, Lee LY, Chen WP, Chen CC, Huang WS, Kuo HC: Protective effects of Hericium erinaceus mycelium and its isolated erinacine A against ischemiainjury-induced neuronal cell death via the inhibition of iNOS/p38 MAPK and nitrotyrosine. Int J Mol Sci 2014;15:15073-15089.

11 Kuo HC, Lu CC, Shen CH, Tung SY, Hsieh MC, Lee KC, Lee LY, Chen CC, Teng CC, Huang WS, Chen TC, Lee KF: Hericium erinaceus mycelium and its isolated erinacine A protection from MPTP-induced neurotoxicity through the ER stress, triggering an apoptosis cascade. J Transl Med 2016;14:78.

12 Lu C-C, Huang W-S, Lee K-F, Lee K-C, Hsieh M-C, Huang C-Y, Lee L-Y, Lee B-O, Teng C-C, Shen C-H, Tung S-Y, Kuo H-C: Inhibitory effect of Erinacines A on the growth of DLD-1 colorectal cancer cells is induced by generation of reactive oxygen species and activation of p70S6K and p21. J Funct Foods 2016;21:474-484. 


\section{Cellular Physiology Cell Physiol Biochem 2017;43:195-208

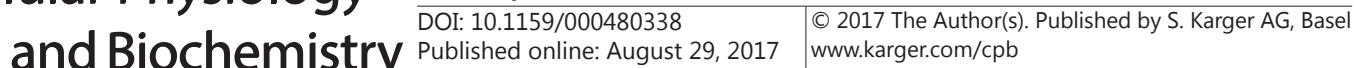

13 Lee KC, Kuo HC, Shen CH, Lu CC, Huang WS, Hsieh MC, Huang CY, Kuo YH, Hsieh YY, Teng CC, Lee LY, Tung SY: A proteomics approach to identifying novel protein targets involved in erinacine A-mediated inhibition of colorectal cancer cells' aggressiveness. J Cell Mol Med 2017;21:588-599.

14 Zheng L, Wang L, Ajani J, Xie K: Molecular basis of gastric cancer development and progression. Gastric Cancer 2004; 7:61-77.

15 Yang L: Incidence and mortality of gastric cancer in China. World J Gastroenterol 2006;12:17-20.

-16 Luo X, Budihardjo I, Zou H, Slaughter C, Wang X: Bid, a Bcl2 interacting protein, mediates cytochrome c release from mitochondria in response to activation of cell surface death receptors. Cell 1998;94:481-490.

-17 Desagher S, Osen-Sand A, Nichols A, Eskes R, Montessuit S, Lauper S, Maundrell K, Antonsson B, Martinou JC: Bid-induced conformational change of Bax is responsible for mitochondrial cytochrome c release during apoptosis. J Cell Biol 1999;144:891-901.

18 Li-Weber M: Targeting apoptosis pathways in cancer by Chinese medicine. Cancer Lett 2013;332:304-312.

-19 Huang WS, Kuo YH, Kuo HC, Hsieh MC, Huang CY, Lee KC, Lee KF, Shen CH, Tung SY, Teng CC: CIL-102Induced Cell Cycle Arrest and Apoptosis in Colorectal Cancer Cells via Upregulation of p21 and GADD45. PLoS One 2017;12:e0168989.

20 Kallergi G, Agelaki S, Markomanolaki H, Georgoulias V, Stournaras C: Activation of FAK/PI3K/Rac1 signaling controls actin reorganization and inhibits cell motility in human cancer cells. Cell Physiol Biochem 2007;20:977-986.

21 Loo G: Redox-sensitive mechanisms of phytochemical-mediated inhibition of cancer cell proliferation (review). J Nutr Biochem 2003;14:64-73.

-22 Papadopoulou N, Charalampopoulos I, Alevizopoulos K, Gravanis A, Stournaras C: Rho/ROCK/actin signaling regulates membrane androgen receptor induced apoptosis in prostate cancer cells. Exp Cell Res 2008;314:3162-3174.

-23 Tseng TH, Lin WL, Chen ZH, Lee YJ, Shie MS, Lee KF, Shen CH, Kuo HC: Moniliformediquinone as a potential therapeutic agent, inactivation of hepatic stellate cell and inhibition of liver fibrosis in vivo. J Transl Med $2016 ; 14: 263$.

24 Huang WS, Kuo YH, Chin CC, Wang JY, Yu HR, Sheen JM, Tung SY, Shen CH, Chen TC, Sung ML, Liang HF, Kuo HC: Proteomic analysis of the effects of baicalein on colorectal cancer cells. Proteomics 2012;12:810-819.

25 Mhawech P: 14-3-3 proteins--an update. Cell Res 2005;15:228-236.

26 Ding A, Zhao W, Shi X, Yao R, Zhou F, Yue L, Liu S, Qiu W: Impact of NPM, TFF3 and TACC1 on the prognosis of patients with primary gastric cancer. PLoS One 2013;8:e82136.

27 Luk SC, Siu SW, Lai CK, Wu YJ, Pang SF: Cell Cycle Arrest by a Natural Product via G2/M Checkpoint. Int J Med Sci 2005;2:64-69.

28 Xia P, Zhou J, Song X, Wu B, Liu X, Li D, Zhang S, Wang Z, Yu H, Ward T, Zhang J, Li Y, Wang X, Chen Y, Guo Z, Yao X: Aurora A orchestrates entosis by regulating a dynamic MCAK-TIP150 interaction. J Mol Cell Biol 2014;6:240-254.

29 Thomas GM, Huganir RL: MAPK cascade signalling and synaptic plasticity. Nat Rev Neurosci 2004;5:173183.

30 Johannessen M, Delghandi MP, Moens U: What turns CREB on? Cell Signal 2004;16:1211-1227.

-31 Jiang K, Wang J, Liu J, Ward T, Wordeman L, Davidson A, Wang F, Yao X: TIP150 interacts with and targets MCAK at the microtubule plus ends. EMBO Rep 2009;10:857-865.

32 Dicken BJ, Bigam DL, Cass C, Mackey JR, Joy AA, Hamilton SM: Gastric adenocarcinoma: review and considerations for future directions. Ann Surg 2005;241:27-39.

-33 Xian X, Huang L, Zhang B, Wu C, Cui J, Wang Z: WIN 55,212-2 Inhibits the Epithelial Mesenchymal Transition of Gastric Cancer Cells via COX-2 Signals. Cell Physiol Biochem 2016;39:2149-2157.

-34 Gao W, Xua J, Wang F, Zhang L, Peng R, Zhu Y, Tang Q Wu J: Mitochondrial Proteomics Approach Reveals Voltage-Dependent Anion Channel 1 (VDAC1) as a Potential Biomarker of Gastric Cancer. Cell Physiol Biochem 2015;37:2339-2354.

35 Zan X, Cui F, Li Y, Yang Y, Wu D, Sun W, Ping L: Hericium erinaceus polysaccharide-protein HEG-5 inhibits SGC-7901 cell growth via cell cycle arrest and apoptosis. Int J Biol Macromol 2015;76:242-253.

-36 Caprioli G, Logrippo S, Cahill MG, James KJ: High-performance liquid chromatography LTQ-Orbitrap mass spectrometry method for tomatidine and non-target metabolites quantification in organic and normal tomatoes. Int J Food Sci Nutr 2014;65:942-947. 


\section{Cellular Physiology Cell Physiol Biochem 2017;43:195-208

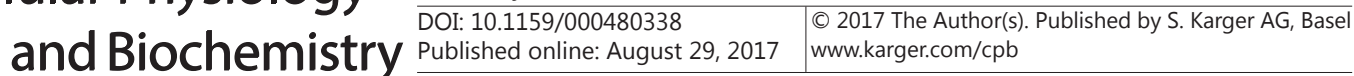 \\ Kuo et al.: Erinacine A Induces Apoptosis and Inhibition of Metastasis in Gastric Cancer}

-37 Li Y, Sun Z, Liu K, Qiu W, Yao R, Feng T, Xin C, Yue L: Prognostic significance of the co-expression of nucleophosmin and trefoil factor 3 in postoperative gastric cancer patients. Mol Clin Oncol 2014;2:10551061.

38 Tsukamoto Y, Nakada C, Noguchi T, Tanigawa M, Nguyen LT, Uchida T, Hijiya N, Matsuura K, Fujioka T, Seto M, Moriyama M: MicroRNA-375 is downregulated in gastric carcinomas and regulates cell survival by targeting PDK1 and 14-3-3zeta. Cancer Res 2010;70:2339-2349.

-39 Gu S, Kounenidakis M, Schmidt EM, Deshpande D, Alkahtani S, Alarifi S, Foller M, Alevizopoulos K, Lang F, Stournaras C: Rapid activation of FAK/mTOR/p70S6K/PAK1-signaling controls the early testosteroneinduced actin reorganization in colon cancer cells. Cell Signal 2013;25:66-73.

40 Papakonstanti EA, Kampa M, Castanas E, Stournaras C: A rapid, nongenomic, signaling pathway regulates the actin reorganization induced by activation of membrane testosterone receptors. Mol Endocrinol 2003;17:870-881.

41 Gu S, Honisch S, Kounenidakis M, Alkahtani S, Alarifi S, Alevizopoulos K, Stournaras C, Lang F: Membrane androgen receptor down-regulates c-src-activity and beta-catenin transcription and triggers GSK-3betaphosphorylation in colon tumor cells. Cell Physiol Biochem 2014;34:1402-1412.

42 Stournaras C, Gravanis A, Margioris AN, Lang F: The actin cytoskeleton in rapid steroid hormone actions. Cytoskeleton (Hoboken) 2014;71:285-293.

43 Anagnostopoulou V, Pediaditakis I, Alkahtani S, Alarifi SA, Schmidt EM, Lang F, Gravanis A, Charalampopoulos I, Stournaras C: Differential effects of dehydroepiandrosterone and testosterone in prostate and colon cancer cell apoptosis: the role of nerve growth factor (NGF) receptors. Endocrinology 2013;154:2446-2456. 